\section{Sífilis materna e congênita: ainda um desafio}

\author{
Maternal and congenital syphilis: a persistent \\ challenge
}

Sífilis materna y congénita: un desafío

${ }^{1}$ Secretaria de Estado de Saúde do Distrito Federal, Brasília, Brasil.

2 Faculdade de Medicina de Botucatu, Universidade Estadual Paulista Júlio de Mesquita Filho, Botucatu, Brasil.

Correspondência D. M. S. Magalhães Secretaria de Estado de Saúde do Distrito Federal. SMHN Quadra 101 Área Especial Asa Norte, Brasília, DF 71000-000, Brasil. danymendes@yahoo.com.br

\section{Abstract}

This descriptive study collected socio-demographic, obstetric, and diagnostic and treatment-related data from pregnant and postpartum women and their partners, for the 67 pregnant or postpartum women reported with syphilis to the National System of Diseases of Notification, users of public maternity hospitals in the Federal District of Brazil from 2009 to 2010. Data on clinical and laboratory followup of the newborn were obtained from the hospital patient charts, compulsory notification forms, and Infant Health Cards. Of the pregnant women, $41.8 \%$ were adequately treated; the main reason for inadequate treatment was the absence (83.6\%) or inadequate treatment of the partner (88.1\%). More than a third required repeat treatment at the maternity hospital due to lack of documentation of treatment during the prenatal period. Of the newborns diagnosed with congenital syphilis, $48 \%$ received radiographic investigation, $42 \%$ received a spinal tap, and $36 \%$ failed to receive any kind of intervention. Thus, the quality of prenatal care was insufficient to guarantee the control of congenital syphilis and achieve the goal of reducing incidence of the disease.

Congenital Syphilis; Prenatal Care; Maternal and Child Health
Daniela Mendes dos Santos Magalhães 1,2 Inês Aparecida Laudares Kawaguchi 1,2 Adriano Dias 2 Iracema de Mattos Paranhos Calderon 2

\section{Resumo}

Este estudo descritivo coletou informações sociodemográficas, obstétricas e relacionadas ao diagnóstico e tratamento da gestante/puérpera e parceiro das 67 gestantes/puérperas notificadas no Sistema Nacional de Agravos de Notificação, usuárias de maternidades públicas do Distrito Federal, Brasil, entre 2009 e 2010. As informações do acompanhamento clínico e laboratorial recebido pela criança vieram do prontuário médico hospitalar, fichas de notificação compulsória e Cartão da Criança. Das gestantes, 41,8\% foram adequadamente tratadas, o principal motivo para a inadequação foi a ausência $(83,6 \%)$ ou inadequação do tratamento do parceiro $(88,1 \%)$. Mais de um terço necessitou de novo tratamento na maternidade por falta de documentação terapêutica no pré-natal. Dos recém-nascidos com sífilis congênita, 48\% fizeram estudo radiográfico, 42\% passaram por punção liquórica e $36 \%$ deles não receberam qualquer tipo de intervenção. Nota-se, assim, que a qualidade do pré-natal recebido pela gestante não é suficiente para garantir o controle da sífilis congênita e o alcance da meta de incidência da doença.

Sífilis Congênita; Cuidado Pré-Natal; Saúde Materno-Infantil 


\section{Introdução}

As doenças sexualmente transmissíveis (DST) constituem um sério problema de saúde pública que acarreta danos sociais, econômicos e sanitários de grande repercussão às populações, especialmente entre mulheres e crianças 1 .

Entre as DST, a sífilis merece destaque. Doença infecciosa e sistêmica, de abrangência mundial e evolução crônica causada pelo Treponema pallidum, tem o homem como único hospedeiro, transmissor e reservatório. Sua transmissão pode ocorrer de forma sexual ou vertical, é mais frequente em grandes centros urbanos e afeta igualmente todas as camadas sociais 2. Ainda se associam à ocorrência de sífilis o baixo nível socioeconômico, coinfecção por HIV, uso de drogas, gravidez na adolescência, história de natimortalidade, comportamento sexual de risco, migração para grandes centros urbanos, acesso limitado aos cuidados de saúde $3,4,5$ e o não tratamento do parceiro infectado 6,7.

O Ministério da Saúde do Brasil lançou, em 1993, o projeto de eliminação da sífilis congênita, em consonância com a proposta de controle do agravo nas Américas, formulado pela Organização Pan-Americana da Saúde (OPAS) e Organização Mundial da Saúde (OMS). Nessa iniciativa, foi definida como meta a redução da incidência a valores menores ou iguais a um caso por mil nascidos vivos. No entanto, no Brasil, foram registrados em 2005, 5.792 casos de sífilis congênita em menores de um ano, refletindo incidência média de 1,9 casos para cada mil nascidos vivos, variando entre as regiões de residência 3 .

Informes oficiais da OMS indicam que, por ano, ocorrem cerca de 12 milhões de novos casos na população adulta em todo mundo, em grande parte em países em desenvolvimento 8,9 No Brasil, estima-se que a prevalência média de sífilis em parturientes varie entre $1,4 \%$ e $2,8 \%$, com uma taxa de transmissão vertical em torno de $25 \% 2,10$.

No Distrito Federal, no período entre 2007 e 2008, foram registrados 186 casos de sífilis em gestantes, gerando um coeficiente de detecção de cerca de 20 casos por 10 mil nascidos vivos, de acordo com dados do Sistema de Informações de Agravos de Notificação da Secretaria de Estado de Saúde do Distrito Federal (SINAN/SES/ DF). Acredita-se, contudo, que tais dados possam estar subestimados em razão da subnotificação, que é realidade em muitos locais 11,12,13.

A qualidade da assistência à gestação e parto é um importante determinante na redução das taxas de transmissão vertical da sífilis e o controle da doença tem como fundamento a triagem sorológica e o tratamento adequado de gestantes e parceiros 14

O Ministério da Saúde preconiza, também, uma série de rotinas diagnósticas e protocolos de atendimento a serem observados no seguimento de crianças nascidas de mães que tiveram diagnóstico desífilisnagestação, parto ou puerpério 10. A conduta a ser adotada baseia-se em quatro aspectos: diagnóstico e adequação do tratamento para sífilis, evidência clínica, laboratorial e radiográfica da sífilis no recém-nascido e comparação da titulação do teste Venereal Disease Research Laboratory (VDRL) materno com o teste do concepto na ocasião do parto 10 . À exceção dos recém-nascidos assintomáticos, com VDRL não reagente e nascidos de mães adequadamente tratadas, todos os demais recém-nascidos com diagnóstico materno de sífilis devem ser submetidos aos procedimentos de rastreamento na maternidade.

Dessa forma, os objetivos deste estudo foram: estabelecer o perfil das gestantes com VDRL reagente acompanhadas em maternidades públicas do Distrito Federal, Brasil; dos recém-nascidos nascidos de mães com sífilis que apresentaram sinais clínicos da doença congênita e verificar a conduta clínica de acordo com as normas preconizadas pelo Ministério da Saúde.

\section{Método}

Trata-se de um estudo descritivo, realizado em maternidades públicas de hospitais regionais que integram a rede assistencial da Secretaria de Estado de Saúde do Distrito Federal (SES/DF) em nível secundário de assistência, sendo duas localizadas na cidade de Brasília (Asa Sul e Asa Norte) e três em Regiões Administrativas do Distrito Federal (Gama, Taguatinga e Ceilândia), no período de novembro de 2009 a dezembro de 2010. Essas maternidades foram escolhidas por apresentarem maiores coeficientes de detecção de sífilis em anos anteriores e maior número de partos. Em relação ao número de casos por 100 mil nascidos vivos, os serviços apresentaram coeficientes de detecção de 376/100 mil nascidos vivos no Hospital Regional da Asa Norte, 378 casos/100 mil nascidos vivos no Hospital Regional de Taguatinga, 506 casos/100 mil nascidos vivos no Hospital Regional da Asa Sul, 163 casos/100 mil nascidos vivos no Hospital Regional da Ceilândia e 65 casos/100 mil nascidos vivos no Hospital Regional do Gama. Esses hospitais foram responsáveis, no ano de 2010 , por $58,7 \%$ dos partos realizados na SES/DF.

A população foi constituída por setenta gestantes VDRL reagente em qualquer momento 
da gestação e/ou na ocasião do parto, usuárias dessas maternidades e notificadas no SINAN. Não foram incluídas na amostra gestantes não residentes no Distrito Federal, visto que esse é o critério adotado pela Diretoria de Vigilância Epidemiológica (DIVEP) da SES/DF para a contagem de casos locais.

A coleta de dados foi realizada em duas etapas: busca ativa das fichas de notificação junto aos Núcleos de Vigilância Epidemiológica dos hospitais regionais que fizeram parte deste estudo e junto às gestantes/puérperas, durante a internação ou após o parto (em até seis meses) por contato telefônico e/ou visita domiciliar.

As informações foram coletadas e registradas em instrumento que continha perguntas sobre aspectos sociodemográficos (idade, escolaridade, renda), obstétricos (parição, número de abortamentos, histórico de pré-natal em gestações anteriores) e epidemiológicos da sífilis da gestante (triagem sorológica, momento do diagnóstico, tratamento) e do seu parceiro (idade, escolaridade, tratamento concomitante). As informações referentes ao acompanhamento clínico e laboratorial recebido pela criança foram complementadas com dados do prontuário médico hospitalar, fichas de notificação compulsória da sífilis, Cartão da Gestante e Cartão da Criança (quando disponível).

Todas as mulheres foram convidadas a participar e, após serem esclarecidas sobre os objetivos e metodologia da pesquisa e terem concordado em dela participar, assinando o Termo de Consentimento Livre e Esclarecido, foram incluídas no estudo. A pesquisa foi aprovada pelo Comitê de Ética em Pesquisa da SES/DF sob o parecer número 425/2009, de acordo com a $R e$ solução no 196/96 do Conselho Nacional de Saúde (CNS) e suas complementares.

Para este trabalho, foi considerado tratamento materno adequado para sífilis todo tratamento completo de acordo com a fase da doença, realizado com penicilina, finalizado até trinta dias antes do parto e com tratamento concomitante do parceiro, de acordo com as normas do Ministério da Saúde para a prevenção da transmissão vertical da sífilis.

A classificação, a conduta clínica e o esquema de tratamento dos recém-nascidos consideraram o diagnóstico e o tratamento da sífilis materna, a evidência clínica, laboratorial ou radiológica da doença no recém-nascido e a comparação das titulações do VDRL materno e do recém-nascido na ocasião do parto. Obedeceram, ainda, às categorias determinadas pelo Ministério da Saúde em Al (presença de alterações clínicas e/ou sorológicas e/ou radiológicas, mas não liquóricas), A2 (presença de alterações liquóricas) e A3 (ausência de alterações clínicas, radiológicas e/ou liquóricas, e sorologia negativa no recém-nascido), com tratamentos distintos em relação à posologia da penicilina.

A pesquisa recebeu financiamento da Fundação de Ensino e Pesquisa em Ciências da Saúde (FEPECS) pelo edital de fomento a pesquisa no 02 de 18 de fevereiro de 2010.

A tabulação e a análise descritiva dos dados foram realizadas utilizando o programa IBM/ SPSS Statistics versão 19.0 (IBM, Nova York, Estados Unidos).

\section{Resultados}

Durante o período de coleta de dados foram identificadas setenta gestantes com VDRL reagente no parto ou durante o pré-natal. Três foram excluídas: uma por recusa em participar, uma por impossibilidade de contato e uma por se encontrar em situação prisional no momento da avaliação. Logo, a amostra final para este estudo foi de 67 gestantes/puérperas.

A média de idade das entrevistadas foi de 28,1 anos, com maior proporção na faixa etária entre 19 a 35 anos (53,7\%). A Tabela 1 mostra a distribuição das gestantes/puérperas de acordo com as variáveis sociodemográficas e antecedentes obstétricos. Em relação à escolaridade, 43 (64,2\%) gestantes/puérperas declararam possuir o Ensino Fundamental completo e 54 (80,6\%) possuíam renda familiar de até quatro salários mínimos.

Das 67 mulheres, 15 eram gestantes e entre elas, três tiveram como desfecho da gestação o abortamento, enquanto 52 eram puérperas, entre as quais duas tiveram natimorto. Quando questionados os antecedentes obstétricos, 54 $(80,6 \%)$ gestantes/puérperas referiram mais de uma gestação e $46(68,7 \%)$ referiram ter realizado pré-natal nas gestações anteriores.

Na Tabela 2, encontram-se as variáveis relacionadas à gestação atual. A flutuação no número de observações para as variáveis tipo de parto $(n=52)$ e início do pré-natal em trimestre $(n=61)$ deveu-se ao fato de 15 mulheres não terem parido no momento da abordagem e 06 não tinham realizado nenhuma consulta pré-natal.

Das gestantes/puérperas, 06 não realizaram o pré-natal $(9 \%), 33(49,3 \%)$ realizaram mais de seis consultas e $34(50,7 \%)$ iniciaram o pré-natal ainda no primeiro trimestre. Do total, 28 gestantes/puérperas foram adequadamente tratadas $(41,8 \%)$ e o principal motivo para a inadequação do tratamento foi a falta $(83,6 \%)$ e/ou inadequação do tratamento do parceiro $(88,1 \%)$. Ainda, 25 gestantes $(37,3 \%)$ necessitaram de novo 
Tabela 1

Distribuição das gestantes de acordo com as variáveis sociodemográficas e antecedentes obstétricos. Brasília, Brasil, 2010.

\begin{tabular}{|c|c|c|c|}
\hline Variáveis & $\mathbf{n}$ & $\%$ & $\%$ acumulado \\
\hline \multicolumn{4}{|l|}{ Faixa etária (anos) } \\
\hline$<19$ & 5 & 7,5 & 7,5 \\
\hline $19-35$ & 36 & 53,7 & 61,2 \\
\hline$>35$ & 26 & 38,8 & 100,0 \\
\hline \multicolumn{4}{|l|}{ Escolaridade } \\
\hline Nenhuma & 1 & 1,5 & 1,5 \\
\hline Ensino Fundamental & 43 & 64,2 & 65,7 \\
\hline Ensino Médio & 21 & 31,3 & 97,0 \\
\hline Ensino Superior & 1 & 1,5 & 98,5 \\
\hline Não informado & 1 & 1,5 & 100,0 \\
\hline \multicolumn{4}{|c|}{ Renda familiar (salários mínimos) } \\
\hline$<1$ & 3 & 4,5 & 4,5 \\
\hline $1-4$ & 54 & 80,6 & 85,1 \\
\hline$>4$ & 10 & 14,9 & 100,0 \\
\hline \multicolumn{4}{|l|}{ Mora com o parceiro } \\
\hline Sim & 29 & 43,3 & 43,3 \\
\hline Não & 38 & 56,7 & 100,0 \\
\hline \multicolumn{4}{|l|}{ Número de gestações } \\
\hline 1 & 13 & 19,4 & 19,4 \\
\hline 2 ou mais & 54 & 80,6 & 100,0 \\
\hline \multicolumn{4}{|l|}{ Número de partos } \\
\hline Nenhum & 16 & 23,9 & 23,9 \\
\hline 1 & 25 & 37,3 & 61,2 \\
\hline 2 ou mais & 26 & 38,8 & 100,0 \\
\hline \multicolumn{4}{|l|}{ Número de abortamentos } \\
\hline Nenhum & 47 & 70,1 & 70,1 \\
\hline 1 & 18 & 26,9 & 97,0 \\
\hline 2 ou mais & 2 & 3,0 & 100,0 \\
\hline \multicolumn{4}{|c|}{ Número de natimortos em gestações anteriores } \\
\hline Nenhum & 63 & 94,0 & 94,0 \\
\hline 1 & 3 & 4,5 & 98,5 \\
\hline 2 ou mais & 1 & 1,5 & 100,0 \\
\hline \multicolumn{4}{|c|}{ Realizou pré-natal nas outras gestações $(n=56)$ * } \\
\hline Sim & 50 & 89,3 & 89,3 \\
\hline Não & 6 & 10,7 & 100,0 \\
\hline
\end{tabular}

* 11 mulheres eram primigestas e, portanto, sem pré-natal anterior.

tratamento no momento do parto por falta de documentação e/ou documentação incompleta do tratamento realizado no pré-natal, e uma gestante por ter aumento do título do VDRL no período. Das gestantes/puérperas, $40(59,7 \%)$ foram informadas do diagnóstico de sífilis no pré-natal.

Entre cinquenta recém-nascidos, 3 (6\%) apresentaram ao nascimento sinais clínicos de infecção congênita por sífilis. 
Tabela 2

Distribuição das gestantes de acordo com as variáveis da gestação atual. Brasília, Brasil, 2010.

\begin{tabular}{|c|c|c|c|}
\hline Variáveis & n & $\%$ & $\%$ acumulado \\
\hline \multicolumn{4}{|l|}{ Uso de métodos contraceptivos } \\
\hline Sim & 14 & 20,9 & 20,9 \\
\hline Não & 53 & 79,1 & 100,0 \\
\hline \multicolumn{4}{|l|}{ Realizou pré-natal } \\
\hline Sim & 61 & 91,0 & 91,0 \\
\hline Não & 6 & 9,0 & 100,0 \\
\hline \multicolumn{4}{|l|}{ Número de consultas } \\
\hline Nenhuma & 6 & 9,0 & 9,0 \\
\hline $1-5$ & 28 & 41,8 & 50,7 \\
\hline$>6$ & 33 & 49,3 & 100,0 \\
\hline \multicolumn{4}{|c|}{ Início do pré-natal (em trimestre, $n=61)$ * } \\
\hline $1 \underline{0}$ & 34 & 55,7 & 55,7 \\
\hline 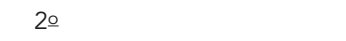 & 20 & 32,8 & 88,5 \\
\hline 3으 & 7 & 11,5 & 100,0 \\
\hline \multicolumn{4}{|l|}{ Diagnóstico de sífilis no pré-natal } \\
\hline Sim & 52 & 77,6 & 77,6 \\
\hline Não & 15 & 22,4 & 100,0 \\
\hline \multicolumn{4}{|l|}{ Realizou teste treponêmico } \\
\hline $\operatorname{Sim}$ & 47 & 70,1 & 70,1 \\
\hline Não & 18 & 26,9 & 97,0 \\
\hline Não Informado & 2 & 3,0 & 100,0 \\
\hline \multicolumn{4}{|l|}{ Tratamento realizado para sífilis } \\
\hline Esquema sífilis primária & 25 & 37,3 & 37,3 \\
\hline Esquema sífilis secundária & 3 & 4,5 & 41,8 \\
\hline Esquema sífilis terciária & 25 & 37,3 & 79,1 \\
\hline Não realizado & 11 & 16,4 & 95,5 \\
\hline Não informado & 1 & 1,5 & 97,0 \\
\hline Outro esquema & 2 & 3,0 & 100,0 \\
\hline \multicolumn{4}{|c|}{ Término do tratamento até trinta dias antes do parto } \\
\hline Sim & 33 & 49,2 & 49,2 \\
\hline Não & 30 & 44,8 & 94,0 \\
\hline Não informado & 4 & 6,0 & 100,0 \\
\hline \multicolumn{4}{|l|}{ Tratamento recebido } \\
\hline Adequado & 28 & 41,8 & 41,8 \\
\hline Não adequado & 39 & 58,2 & 100,0 \\
\hline \multicolumn{4}{|c|}{ Momento em que foi informada sobre o diagnóstico } \\
\hline Pré-natal & 40 & 59,7 & 59,7 \\
\hline Parto & 8 & 11,9 & 71,6 \\
\hline Puerpério & 3 & 4,5 & 76,1 \\
\hline Tinha conhecimento prévio & 14 & 20,9 & 97,0 \\
\hline Após aborto & 2 & 3,0 & 100,0 \\
\hline \multicolumn{4}{|l|}{ Tipo de parto $(n=52) \star \star$} \\
\hline Vaginal & 34 & 65,4 & 65,4 \\
\hline Cirúrgico & 18 & 34,6 & 100,0 \\
\hline \multicolumn{4}{|c|}{ Cartão da Gestante devidamente preenchido com } \\
\hline \multicolumn{4}{|l|}{ informações sobre a sífilis } \\
\hline Sim & 33 & 49,3 & 49,3 \\
\hline Não & 25 & 37,3 & 86,6 \\
\hline Não possui cartão & 9 & 13,4 & 100,0 \\
\hline
\end{tabular}

* 6 mulheres não tinham realizado nenhuma consulta pré-natal;

** 15 mulheres eram nulíparas. 
Distribuição dos recém-nascidos por características ao nascer, conduta inicial para detecção de sífilis congênita e tratamento instituído. Brasília, Brasil, 2010.

\begin{tabular}{|c|c|c|c|}
\hline Variáveis & $\mathrm{n}$ & $\%$ & $\%$ acumulado \\
\hline \multicolumn{4}{|l|}{ Sexo } \\
\hline Masculino & 26 & 52,0 & 52,0 \\
\hline Feminino & 24 & 48,0 & 100,0 \\
\hline \multicolumn{4}{|l|}{ Idade gestacional (semanas) } \\
\hline$<37$ & 11 & 22,0 & 22,0 \\
\hline 37 ou mais & 39 & 78,0 & 100,0 \\
\hline \multicolumn{4}{|l|}{ Peso ao nascer (g) } \\
\hline$<2.500$ & 6 & 12,0 & 12,0 \\
\hline 2.500 ou mais & 44 & 88,0 & 100,0 \\
\hline \multicolumn{4}{|c|}{ Sinais clínicos de infecção congênita } \\
\hline Sim & 3 & 6,0 & 6,0 \\
\hline Não & 45 & 90,0 & 96,0 \\
\hline Ignorado & 2 & 4,0 & 100,0 \\
\hline \multicolumn{4}{|c|}{ Exames realizados no rastreamento } \\
\hline VDRL de sangue periférico & 46 & 92,0 & - \\
\hline Hemograma & 49 & 98,0 & - \\
\hline Raio-x de ossos longos & 24 & 48,0 & - \\
\hline Punção lombar & 21 & 42,0 & - \\
\hline \multicolumn{4}{|l|}{ Tratamento instituído * } \\
\hline A1 & 5 & 10,0 & 10,0 \\
\hline $\mathrm{A} 2$ & 11 & 22,0 & 32,0 \\
\hline A3 & 16 & 32,0 & 64,0 \\
\hline Não realizado & 18 & 36,0 & 100,0 \\
\hline
\end{tabular}

*A1: presença de alterações clínicas e/ou sorológicas e/ou radiológicas, mas não liquóricas - Penicilina G Cristalina 100.000Ul/kg/dia, EV, duas doses/dia (menos que sete dias de vida) ou três doses/dia (se mais de sete dias), por 10 dias; ou Penicilina G Procaína - 50.000UI/kg/dia, IM, por 10 dias; A2: presença de alterações liquóricas - Penicilina G Cristalina 150.000Ul/kg/dia, EV, duas doses/dia (menos de sete dias de vida) ou três doses/dia (se mais de sete dias), por 14 dias; A3: ausência de alterações clínicas, radiológicas e/ou liquóricas, e a sorologia negativa no recém-nascido - Penicilina G Benzatina, IM, dose única, $50.000 \mathrm{Ul} / \mathrm{kg}$.

A Tabela 3 mostra a distribuição dos recémnascidos por características ao nascer, exames realizados no rastreamento para sífilis congênita e tratamento instituído por ocasião do parto.

\section{Discussão}

Em 2006, com o objetivo de fortalecer a gestão compartilhada entre as diversas esferas de governo, o CNS aprovou o Pacto pela Saúde. Por esse instrumento, estados e municípios se comprometem a desenvolver as ações necessárias para o cumprimento de metas adequadas à realidade local, de modo que suas prioridades pudessem agregar-se à agenda nacional.

Um dos seus eixos (Pacto pela Vida) aponta para a redução da mortalidade materna e infantil como uma das prioridades básicas, indicando a redução das taxas de transmissão vertical do HIV e da sífilis como estratégias para sua execução.

Embora a sífilis seja uma doença conhecida há séculos e que tenha agente etiológico bem definido, tratamento eficaz e de baixo custo, estabelecido desde 1943, é grande a proporção de gestantes infectadas que não são sujeitas às ações terapêuticas recomendadas pelo o Programa de Humanização do Pré-Natal e Nascimento em relação ao controle e prevenção da transmissão vertical 15,16 .

Os resultados desta investigação indicaram que a sífilis no período gestacional ocorre em mulheres jovens (média de idade de 28 anos), com pouca escolaridade e baixa renda. Sá et al. 17, em estudo sobre a prevalência e fatores de risco em gestantes de uma maternidade escola no Rio 
de Janeiro no período de 1994 a 1999, observaram correlação entre o VDRL positivo, a idade e baixo grau de instrução materna.

O estudo multicêntrico nacional sobre soroprevalência da sífilis em puérperas realizado no período de 1999 a 2000 com uma amostra de 3.047 mulheres, mostrou que o maior risco para VDRL positivo estava associado, dentre outros fatores, à renda familiar, idade materna e baixa escolaridade 5. Saraceni et al. 18, ao descreverem a mortalidade perinatal por sífilis no Rio de Janeiro nos anos de 1999 e 2002, encontraram características de mães com sífilis semelhantes às encontradas em outros estudos em que o status econômico e a idade materna estavam fortemente associados à maior prevalência da doença 19,20. Em outros dois estudos, realizados em Sergipe e São Paulo, os autores encontraram semelhante faixa etária, mulheres adultas jovens 21,22.

A sífilis em gestantes tem sido relacionada ao baixo nível socioeconômico. Embora não seja uma doença restrita às camadas menos favorecidas, esses resultados sinalizam que pouca escolaridade e baixa renda podem ser marcadores importantes de pouco acesso aos serviços de saúde. Consequentemente, a assistência prénatal inadequada contribui para a persistência da transmissão vertical da sífilis nessa população 23,24,25. Estudo realizado em Recife (Pernambuco) encontrou características maternas semelhantes às encontradas nesta pesquisa e confirma a influência da condição de vida na determinação da sífilis congênita, mostrando maior ocorrência do agravo no segmento mais empobrecido da população 26,27 . Viana et al. 28 , em estudo sobre as desigualdades sociais em saúde no Brasil, afirmam que existe uma relação inversamente proporcional entre a taxa de pobreza e a cobertura pré-natal. Realidade também encontrada em outros estudos que expõem as fragilidades na assistência pré-natal relativas ao acesso e à oportunidade de diagnóstico e tratamento de gestantes e parceiros 15,29,30.

A oferta de serviços de assistência pré-natal altera os desfechos das gestações e a sua ausência pode elevar a mortalidade perinatal em até cinco vezes. Em países em desenvolvimento pode diminuir a mortalidade materna em até $26 \% 31$. No Distrito Federal existem 68 Centros de Saúde, uma unidade mista de saúde e dez maternidades; os $10 \%$ de gestantes que afirmaram não ter realizado pré-natal parece indicar uma falha na cobertura. A OPAS considera adequada uma oferta de serviços de assistência pré-natal com frequência mínima de seis consultas 32 . Neste estudo, seis gestantes/puérperas não fizeram nenhuma consulta, 28 tiveram até cinco consultas e pelo menos 3 mulheres que disseram ter realizado alguma consulta de pré-natal não tinham comprovação documental como o Cartão da Gestante, o que demonstra uma inadequação na oferta e na qualidade dos serviços de assistência prénatal.

O impacto de uma oferta adequada de serviços de saúde é mais significativo quando se considera que é a intervenção de mais curto prazo em saúde pública (duração de nove meses), com alta efetividade dos resultados, prevenindo desfechos adversos da gestação e reduzindo os gastos com a assistência ao recém-nascido. Para tanto precisa ser acessível e ter qualidade. A qualidade dos serviços, para Donabedian 33 , pode ser estimada pela análise da estrutura, do processo e do resultado, sendo o processo a melhor forma de investigar o conteúdo da atenção oferecida no primeiro nível da assistência. De acordo com Silveira et al. ${ }^{34}$, os estudos que visam avaliar a qualidade dos serviços são escassos e em sua maioria limitam-se a descrever características da atenção médica como o número de consultas e tipo de parto. No Brasil, Takeda 35 investigou a qualidade desse cuidado pelo emprego de indicadores de adequação como o índice de Kessner, algoritmo baseado no trimestre gestacional em que o cuidado pré-natal se inicia e no número de consultas, ajustado para a idade gestacional, sendo definido por três categorias de utilização: pré-natal adequado, intermediário e inadequado; e a verificação do conteúdo das consultas.

Halpern et al. ${ }^{36}$, em um estudo de coorte realizado em Pelotas (Rio Grande do Sul), demonstraram que apesar da alta cobertura pré-natal no município, existem desigualdades nos cuidados oferecidos às gestantes, em que as de maior risco gestacional apresentaram maior proporção de seguimento pré-natal inadequado ou intermediário, quando comparadas com as de menor risco. Aquelas que não receberam nenhum atendimento antes do parto eram mais pobres, adolescentes ou com idade acima de quarenta anos e tiveram uma incidência de baixo peso ao nascer 2,5 vezes maior do que aquelas do grupo de mães que realizaram cinco ou mais consultas.

À semelhança de outros estudos, a maior parte das gestantes/puérperas relatou acompanhamento pré-natal com início no primeiro trimestre 14,37,38. O início precoce da assistência prénatal não assegurou um desfecho mais favorável, mesmo nas $33(44,9 \%)$ gestantes com mais de seis consultas, uma vez que 39 mulheres $(58,2 \%)$ foram consideradas inadequadamente tratadas. Esses dados revelam que a garantia de um número minino de consultas de pré-natal pode não ser suficiente para assegurar uma assistência de qualidade 21,27,39. 
O principal fator de falha no tratamento da gestante foi a falta e/ou a inadequação do tratamento do parceiro, fato que se assemelha aos achados em outros estudos 19,21,25,40. A inclusão do parceiro no pré-natal tem sido uma importante estratégia para a abordagem do problema 41 e é determinante para a cura eficaz da mãe e, consequentemente, para o fim do agravo.

Pesquisa sobre os casos notificados de sífilis congênita em menores de um ano no período de 1998 a 2008 verificou que a maioria dos casos era de conceptos de mães jovens (entre 20 e 29 anos), com pouca escolaridade, adeptas ao pré-natal, diagnosticadas no período gestacional e cujos parceiros sexuais não aderiram ao tratamento concomitante 41 .

Ainda é bastante disseminada a ideia de que as demandas dos serviços de saúde são destinadas quase que exclusivamente às mulheres, crianças e idosos, fato pode justificar a pouca presença masculina nos serviços de saúde 42 . Ao homem, ainda se associa a desvalorização do autocuidado e a preocupação incipiente com sua condição de saúde 43 . Soma-se a essas percepções centradas no comportamento do sujeito, ainda, a visão das unidades de saúde como a causa da dificuldade de acesso dos homens aos serviços 42 . Nesse caso, sugere-se que os homens sentiriam mais dificuldades em serem atendidos, seja pelo tempo de espera ou por considerar os serviços de saúde como um espaço feminilizado, que no contexto do pré-natal se torna mais evidente, por ser frequentado particularmente por mulheres e composto por equipe profissional predominantemente feminina 43 .

No Brasil, utiliza-se até o momento o teste não treponêmico de floculação do tipo VDRL para a triagem, seguimento terapêutico e controle de cura. A confirmação laboratorial, com testes treponêmicos por aglutinação de partículas (TPHA) ou teste fluorescente por absorção de anticorpos (FTA-Abs) é recomendada, mas não obrigatória, porém sua ausência não deve retardar a conduta 15 .

Em relação à sorologia treponêmica, 18 gestantes/puérperas $(26,9 \%)$ não tiveram a confirmação do diagnóstico pelo FTA-Abs ou TPHA que talvez possa ser justificado pela falta de insumo para a realização do exame durante um período da coleta de dados. A dificuldade de acesso a exames laboratoriais e, consequentemente, ao tratamento contribui para a dificuldade de controle da doença e para a interpretação das sorologias 16 .

A sífilis em gestantes, quando inadequadamente tratada, pode levar a desfechos adversos para o concepto como abortamento, prematuridade e óbito. Estudo retrospectivo com puér- peras VDRL reagentes e que tiveram o óbito fetal como desfecho da gestação apontou que a presença da sífilis materna como a principal causa dessas ocorrências 45 .

Neste estudo, três gestantes tiveram como desfecho de suas gestações o aborto, todavia, a ausência dos laudos histopatológicos não sustenta a associação entre o desfecho e a reatividade materna positiva para a sífilis. Outros estudos já relataram casos de abortos espontâneos, nos primeiro e segundo trimestres, cujos laudos histopatológicos revelaram a presença do T. pallidum no tecido fetal e placenta 46,47 . Neste trabalho, ocorreram dois casos de natimortalidade, sendo um associado à hipertensão gestacional e o outro não foi possível determinar se a causa do óbito estava relacionada à sífilis congênita.

Entre as gestantes/puérperas, $14(20,9 \%)$ declararam conhecimento prévio da doença, o que pode sinalizar um tratamento inadequado naquela ocasião, reinfecção posterior ou cicatriz sorológica. A sífilis na gestante é considerada adequadamente tratada, afastando a possibilidade de infecção congênita, quando a mulher é medicada com penicilina nas doses apropriadas para a fase da infecção, o tratamento é finalizando em pelo menos trinta dias antes do parto e o seu parceiro é tratado concomitantemente com o mesmo esquema terapêutico da gestante 3 . Por mais que trouxessem informações sobre o tratamento da mulher, os dados que constavam tanto no cartão da gestante (quando disponível), quanto nas informações do prontuário, não faziam referência ao tratamento do parceiro. Tal tratamento, quando descrito, constava nas fichas de notificação compulsória da doença.

Cabe ressaltar ainda que, tendo em conta a importância de registros referentes à notificação e ao acompanhamento de casos, que a subnotificação, o preenchimento incompleto e/ou informações conflituosas ou ignoradas foram observadas em parte significativa dos registros consultados neste estudo.

O sub-registro das intercorrências, resultados de exames, tratamentos realizados e fatores de risco em um instrumento especialmente destinado a essa finalidade, como é o Cartão da Gestante, juntamente com a subnotificação de casos de um agravo de notificação compulsória, também demonstra uma deficiência na assistência pré-natal por não utilizar adequadamente um recurso que contempla o cuidado pré-natal.

Uma assistência pré-natal deficiente leva à ocorrência de falhas no tratamento de gestantes com sífilis e decorrentemente pode resultar em um aumento no número de casos da sífilis congênita. O diagnóstico e o tratamento da sífilis congênita são mais complexos que os da sífilis 
materna e envolve o prolongamento da hospitalização bem como a realização de exames mais dispendiosos, o que pode acarretar danos individuais, familiares e custos adicionais ao sistema de saúde.

Todos os recém-nascidos de mães inadequadamente tratadas devem ser submetidos ao exame físico, VDRL, hemograma completo, estudo radiográfico de ossos longos e punção lombar para estudo do liquor 3 .

O esquema terapêutico do recém-nascido depende dos achados clínicos, laboratoriais e radiográficos, e varia desde uma aplicação única de penicilina cristalina até esquemas com doses diárias de penicilina cristalina por dez dias 3,10.

Os resultados deste estudo demonstram desacordo com as recomendações do Ministério da Saúde para o seguimento do recém-nascido com sífilis congênita em uma quantidade significativa de casos. Em relação ao estudo radiográfico, apenas 24 (48\%) crianças realizaram; a punção liquórica foi realizada somente em 22 (42\%). Os exames mais realizados foram o hemograma (98\%) e oVDRL de sangue periférico (92\%). Resultados semelhantes foram encontrados em estudo sobre caracterização epidemiológica da sífilis congênita no Município de Salvador (Bahia) em 200746 e em outro realizado no Rio de Janeiro 49 .

Em alguns prontuários, embora houvesse o registro de solicitação dos exames, os laudos não foram localizados e por isso não foi possível determinar se esses procedimentos foram realizados e não devidamente registrados, fato que reforça a precariedade do serviço em relação à documentação para seguimento posterior de casos, apesar de sua importância.

Ainda com relação ao registro de informações, não foram encontradas anotações a respeito de encaminhamentos à rede de atenção básica para o seguimento ambulatorial e por essa razão não foi possível avaliar o seguimento do recémnascido após a alta da maternidade.

Constatou-se que 18 recém-nascidos (36\%) não receberam qualquer tipo de tratamento, todos com VDRL de sangue periférico não reagente. Tal fato evidencia falta de preparo da equipe multidisciplinar em relação às recomendações oficiais, tendo em vista que o Ministério da Saúde recomenda profilaxia com penicilina G benzatina em dose única inclusiva para os casos nascidos de mães adequadamente tratadas, sem sinais de infecção congênita e VDRL não reagente 3 .

\section{Conclusão}

O estudo indicou que a qualidade do pré-natal recebido pela gestante não é suficiente para garantir o controle da sífilis congênita e o alcance da meta de incidência da doença e que a conduta inicial para detecção e tratamento da sífilis congênita não está de acordo com as diretrizes definidas pelo Ministério da Saúde do Brasil.

Os resultados reforçam que a redução da ocorrência da sífilis no período gestacional e, consequentemente, da sífilis congênita, somente será possível quando a adoção de medidas mais efetivas de prevenção e controle foram sistematicamente aplicadas.

A fixação da mulher no serviço de saúde pela captação precoce, oferta de rotina mínima de exames preconizados pelos protocolos, registros apropriados e garantia de tratamento oportuno e adequado, inclusive de parceiros, com acolhimento e reconhecimento de necessidades, são estratégias para a organização do serviço, melhoria da qualidade e seguimento efetivo dos casos. 


\section{Resumen}

El presente estudio descriptivo está basado en datos sociodemográficos, obstétricos, además se encuentra relacionado con el diagnóstico y tratamiento de la embarazada, así como de su pareja, en un grupo de 67 gestantes inscritas en el Sistema Nacional de Vigilancia Sanitaria, usuarias del servicio de maternidad público en el Distrito Federal, Brasil, a partir de 2009 hasta 2010. La información, seguimiento clínico y de laboratorio recibidos sobre los niños provenían de historiales hospitalarios. Un 41,8\% de las mujeres embarazadas fueron tratadas correctamente, no obstante, la razón principal de la inadecuación en el tratamiento fue la ausencia del mismo en un 83,6\% o el tratamiento inadecuado del compañero en un $88,1 \%$. Más de un tercio tuvo que ser sometida a un nuevo tratamiento en la maternidad, debido a la falta de documentación sobre la terapéutica prenatal recibida. A un $48 \%$ de los recién nacidos con sífilis congénita se les realizó un estudio radiográfico, al $42 \%$ punción lumbar y el $36 \%$ de ellos no recibieron ningún tipo de intervención. Por lo tanto, es evidente que la calidad del servicio prenatal recibido por las mujeres embarazadas no es suficiente para asegurar el control de la sífilis congénita.

Sífilis Congénita; Atención Prenatal; Salud MaternoInfantil

\section{Colaboradores}

D. M. S. Magalhães participou do levantamento e coleta de dados, análise e interpretação dos dados, redação final e apresentação dos resultados. I. A. L. Kawaguchi colaborou no levantamento e coleta de dados, análise e interpretação dos dados e redação final. A. Dias contribuiu na elaboração do desenho, análise dos dados e redação final. I. M. P Calderon colaborou na análise dos dados e redação final do artigo.

\section{Agradecimentos}

À Fundação de Ensino e Pesquisa em Ciências da Saúde (FEPECS/SES/DF) pelo financiamento.

\section{Referências}

1. Valderrama J, Zacarías F, Mazin R. Sífilis materna y sífilis congénita en América Latina: un problema grave de solución sencilla. Rev Panam Salud Pública 2004; 16:211-7.

2. Paez M, Riveros MIR. Situación epidemiológica de la sífilis materna y congênita en el sub sector público a nível nacional, Paraguay - 2000-2004. DST J Bras Doenças Sex Transm 2006; 18:117-23.

3. Secretaria de Vigilância em Saúde, Ministério da Saúde. Protocolo para a prevenção de transmissão vertical de HIV e sífilis - manual de bolso. Brasília: Ministério da Saúde; 2007
4. Carvalho JPP, Belda W, Jorge JC. Sífilis em gestantes atendidas na Agência Central do INAMPS de Florianópolis, Santa Catarina. An Bras Dermatol 1983; 58:67-70.

5. Rodrigues CS, Guimarães MDC; Grupo Nacional de Estudo sobre Súfilis Congênita. Positividade para sífilis em puérperas: ainda um desafio para o Brasil. Rev Panam Salud Pública 2004; 16:168-75.

6. Green T, Talbot MD, Morton RS. The control of syphilis, a contemporary problem: a historical perspective. Sex Transm Infect 2001; 77:214-7. 
7. Fernandes RCSC, Fernandes PGCC, Nakata TY. Análise dos casos de sífilis congênita na maternidade do hospital da sociedade portuguesa de beneficência de Campos, RJ. DST J Bras Doenças Sex Transm 2007; 19:157-61.

8. World Health Organization. Global prevalence and incidence of selected curable sexually transmitted diseases: overview and estimates. Geneva: World Health Organization; 2001.

9. Galban E, Benzaken AS. Situación de la sífilis en 20 países de Latinoamérica y el Caribe: año 2006. DST J Bras Doenças Sex Transm 2007; 19:166-72.

10. Secretaria de Vigilância em Saúde, Ministério da Saúde. Diretrizes para o controle da sífilis congênita. Brasília: Ministério da Saúde; 2005.

11. Araújo EC. Importância do pré-natal na prevenção da sífilis congênita. Rev Para Med 2006; 20:47-51.

12. Milanez H, Amaral E. Por que ainda não conseguimos controlar o problema da sífilis em gestantes e recém-nascidos? Rev Bras Ginecol Obstet 2008; 30:325-7.

13. Araújo MAL, Silva DMA, Silva RM, Gonçalves MLC. Análise da qualidade dos registros nos prontuários de gestantes com exame de VDRL reagente. Rev APS 2008; 11:4-9.

14. Campos ALA, Araújo MAL, Melo SP, Gonçalves MLC. Epidemiologia da sífilis gestacional em Fortaleza, Brasil: um agravo sem controle. Cad Saúde Pública 2010; 26:1747-56.

15. Amaral E. Sífilis na gravidez e óbito fetal: de volta para o futuro. Rev Bras Ginecol Obstet 2012; 34:52-5.

16. World Health Organization. Investment case for eliminating mother-to-child transmission of syphilis: promoting better maternal and child health and stronger health systems. Geneva: World Health Organization; 2012.

17. Sá RAM, Bornia RBG, Cunha AA, Oliveira CA, Rocha GPG, Giordano EB. Sífilis e gravidez: avaliação da prevalência e fatores de risco nas gestantes atendidas na Maternidade Escola - UFRJ. DST J Bras Doenças Sex Transm 2001; 13:6-8.

18. Saraceni V, Guimarães MHFS, Theme Filha MM, Leal MC. Mortalidade perinatal por sífilis congênita: indicador da qualidade da atenção à mulher e à criança. Cad Saúde Pública 2005; 21:1244-50.

19. De Loranzi DRS, Madi JM. Sífilis congênita como indicador da assistência pré-natal. Rev Bras Ginecol Obstet 2001; 23:647-52.

20. Lago EG, Rodrigues LC, Fiori RM, Stein AT. Congenital syphilis: identification of two distint profiles maternal characteristics associated with risk. Sex Transm Dis 2004; 31:33-7.

21. Donalísio MR, Freire JB, Mendes ET. Investigação da sífilis congênita na microrregião de Sumaré, Estado de São Paulo, Brasil - desvelando a fragilidade do cuidado à mulher gestante e ao recém-nascido. Epidemiol Serv Saúde 2007; 16:165-73.

22. Inagaki ADM, Oliveira LAR, Oliveira MFB, Santos RCS, Araújo RM, Alves JAB, et al. Soroprevalência de anticorpos para toxoplasmose, rubéola, citomegalovírus, sífilis e HIV em gestantes sergipanas. Rev Soc Bras Med Trop 2009; 42:532-6.
23. Hildebrand VLPC. Sífilis congênita: fatores asso ciados ao tratamento das gestantes e seus parceiros [Dissertação de Mestrado]. Rio de Janeiro: Escola Nacional de Saúde Pública Sergio Arouca, Fundação Oswaldo Cruz; 2010.

24. Rodrigues CS. Sífilis na gestação e puerpério: oportunidades estratégicas para a sua prevenção e controle no Brasil, 2000 [Tese de Doutorado]. Belo Horizonte: Faculdade de Medicina, Universidade Federal de Minas Gerais; 2005.

25. Figueiró-Filho EA, Gardenal RVC, Assunção LA, Costa GR, Periotto CRL, Vedovatte CA, et al. Sífilis congênita como fator de assistência pré-natal no município de Campo Grande - MS. DST J Bras Doenças Sex Transm 2007; 19:139-43.

26. Ramos Jr. AN, Matida LH, Saraceni V, Veras MASM, Pontes RJS. Control of mother-to-child transmission of infectious diseases in Brazil: progress in HIV/AIDS and failure in congenital syphilis. Cad Saúde Pública 2007; 23 Suppl 3:S370-8.

27. Melo NGDO, Melo Filho DA, Ferreira LOC. Diferenciais intraurbanos de sífilis congênita no Recife, Pernambuco, Brasil (2004-2006). Epidemiol Serv Saúde 2011; 20:213-22.

28. Vianna SM, Nunes A, Santos JR, Barata RB. Medindo as desigualdades sociais em saúde no Brasil uma proposta de monitoramento. Brasília: Organização Pan-Americana da Saúde; 2001.

29. Carvalho VCP, Araújo TVB. Adequação da assistência pré-natal em gestantes atendidas em dois hospitais de referência para gravidez de alto risco do Sistema Único de Saúde, na cidade de Recife, Estado de Pernambuco. Rev Bras Saúde Matern Infant 2007; 7:309-17.

30. Saraceni V, Domingues RMSM, Vellozo V, Lauria LM, Dias MAB, Ratto KMN, et al. Vigilância da sífilis na gravidez. Epidemiol Serv Saúde 2007; 16:10311 .

31. Lima BGC. Abordagem da sífilis como indicador de qualidade da assistência pré-natal em Salvador. Rev Baiana Saúde Pública 2004; 28:9-18.

32. Rede Interagencial de Informações para a Saúde. Indicadores básicos de saúde no Brasil: conceitos e aplicações. Brasília: Organização Pan-Americana da Saúde; 2002.

33. Donabedian A. The quality of care: how can it be assessed? JAMA 1988; 260:1743-8.

34. Silveira DS, Santos IS, Dias-da-Costa JS. Atenção pré-natal na rede básica: uma avaliação da es trutura e do processo. Cad Saúde Pública 2001; 17:131-9.

35. Takeda S. Avaliação de unidade de atenção primária: modificação dos indicadores de saúde e qualidade da atenção [Dissertação de Mestrado] Pelotas: Universidade Federal de Pelotas; 1993.

36. Halpern R, Barros FC, Victora CG, Tomas E. Atenção pré-natal em Pelotas, Rio Grande do Sul, Brasil, 1993. Cad Saúde Pública 1998; 14:487-92.

37. Kilsztajn S, Rossbach A, Carmo MSN, Sugahara GTL. Assistência pré-natal, baixo peso e prematuridade no Estado de São Paulo, 2000. Rev Saúde Pública 2003; 37:303-10. 
38. Lima BGC, Costa MCN, Dourado MIC. Avaliação da qualidade do rastreamento de HIV/AIDS e sífilis na assistência pré-natal. Epidemiol Serv Saúde 2008; 17:124-7.

39. Holanda MTCG, Barreto MA, Machado RMM, Pereira RC. Perfil epidemiológico da sífilis congênita no Município do Natal, Rio Grande do Norte - 2004 a 2007. Epidemiol Serv Saúde 2011; 20:203-12.

40. Trevisan MR, De Loranzi DRS, Araújo NM, Ésber K. Perfil da assistência pré-natal entre usuárias do Sistema Único de Saúde em Caxias do Sul. Rev Bras Ginecol Obstet 2002; 24:293-9.

41. Oliveira DR, Figueiredo MSN. Abordagem conceitual sobre a sífilis na gestação e o tratamento de parceiros sexuais. Enferm Foco (Brasília) 2011 2:108-11.

42. Ministério da Saúde. Curso básico de vigilância epidemiológica em sífilis congênita, sífilis em gestantes, infecção pelo HIV em gestantes e crianças expostas. Brasília: Ministério da Saúde; 2009.

43. Departamento de Ações Programáticas Estratégicas, Secretaria de Atenção à Saúde, Ministério da Saúde. Política Nacional de Atenção Integral à Saúde do Homem: princípios e diretrizes. Brasília: Ministério da Saúde; 2008.
44. Figueiredo W. Assistência à saúde dos homens: um desafio para os serviços de atenção primária. Ciênc Saúde Coletiva 2005; 10:105-9.

45. Nascimento MI, Cunha AA, Guimarães EV, Alvarez FS, Oliveira SRSM, Villas Boas EL. Gestações complicadas por sífilis materna e óbito fetal. Rev Bras Ginecol Obstet 2012; 34:56-62.

46. Vieira AA. Contribuição ao estudo epidemiológico de sífilis congênita no município de CarapicuíbaSP: ainda uma realidade em 2001. DST J Bras Doenças Sex Transm 2005; 17:10-7.

47. Temmerman M, Lopita MI, Sanghvi HC, Sinei SK, Plummer FA, Piot P. The role of maternal syphilis, gonorrhoea and HIV-1 infections in spontaneous abortion. Int J STD AIDS 1992; 3:418-22.

48. Almeida MFG, Pereira SM. Caracterização epidemiológica da sífilis congênita no município de Salvador, Bahia. DST J Bras Doenças Sex Transm 2007; 19:144-56.

49. Saraceni V, Velozzo V, Leal MC, Hartz ZMA. Estudo da confiabilidade do SINAN a partir das campanhas para a eliminação da sífilis congênita no Município do Rio de Janeiro. Rev Bras Epidemiol 2005; 8:419-24.

Recebido em 31/Out/2012

Versão final reapresentada em 18/Jan/2013

Aprovado em 31/Jan/2013 